\title{
Un droit sans émotions. Iram non novit jus: esquisse des rapports entre sciences et droit
}

Alain Papaux

\section{OpenEdition}

\section{Journals}

Édition électronique

URL : http://journals.openedition.org/ress/70

DOI : $10.4000 /$ ress.70

ISSN : 1663-4446

Éditeur

Librairie Droz

Édition imprimée

Date de publication : 1 mai 2009

Pagination : 105-179

ISBN : 978-2-600-01303-1

ISSN : 0048-8046

\section{Référence électronique}

Alain Papaux, « Un droit sans émotions. Iram non novit jus: esquisse des rapports entre sciences et droit », Revue européenne des sciences sociales [En ligne], XLVII-144 | 2009, mis en ligne le 01 mai 2012, consulté le 30 avril 2019. URL : http://journals.openedition.org/ress/70 ; DOI : 10.4000/ress.70 


\begin{abstract}
Alain PAPAUX

\section{UN DROIT SANS ÉMOTIONS IRAM NON NOVIT JUS: ESQUISSE DES RAPPORTS} ENTRE SCIENCES ET DROIT
\end{abstract}

\title{
OUVERTURE
}

A l'audition du mot «émotion» dans un contexte juridique, nos cerveaux occidentaux, à tout le moins romano-canoniques (tradition du droit dit «continental»), pensent - si tant est que l'on puisse affirmer que «le cerveau pense ${ }^{1}-$ crime passionnel: un mari, évidemment, trompé, est submergé par l'émotion, saisit alors quelque objet massif à portée de main et poursuit de son ire l'amant qui se sauvera d'une fenêtre ou trépassera sous les coups.

Dans l'hypothèse la moins favorable pour celui-ci, le mari sera arrêté puis jugé pour crime passionnel. L'émotion s'affiche de la sorte au cœur de l'un des scenarii les plus classiques du droit, à savoir de l'une des fabula ${ }^{2}$ les plus partagées dans la culture occidentale... au reste un peu passé de mode.

En revanche, la référence au droit pénal comme parangon du droit dans l'esprit de bien des non-juristes, et parmi eux nombre de philosophes, conserve toute son importance, redoublant de la sorte les effets déformants de ce procédé métonymique, puisque fondé sur une branche juridique assez volontiers qualifiée en théorie du droit de «sui generis» sur fond de summa divisio droit public/droit privé, dernière dichotomie au reste toujours davantage relativisée. Ce choix de la partie, parmi les plus singulières du droit, chargée de refléter le fonctionnement ou l'esprit du tout - en l'occurrence le tout «droit»- est d'autant plus malheureux pour notre sujet que les émotions y sont généreusement présentes et opérantes, médiation nettement plus réduite dans les autres branches: l'émotionnel ne se plaide guère en droit commercial ou administratif par exemple.

Le classicisme de l'espèce «crime passionnel» ne laisse pourtant d'être troublant en ce que l'assomption même de cette émotion en et par le droit a conduit à des résultats opposés, voire contradictoires. La jalousie - irrépressible - du mâle le submergeant doit-elle être qualifiée de circonstance atténuante - combien il apparaissait légitime qu'un mari bafoué s'octroie quelques onces de justice privée à la moralité hautement souhaitable, pensait-on alors - ou au contraire de circons-

1 Allusion à la controverse fameuse entre J.-P. Changeux et P. Ricoeur ayant donné lieu à un ouvrage synthétisant leur dialogue, mettant en exergue maintes confusions entre ontologie et épistémologie que Ricoeur détisse patiemment: Ce qui nous fait penser. La Nature et la Règle, Odile Jacob (1998), 2000.

2 Au sens large de fabula: une séquence d'actions selon U. Eco, De Superman au Surhomme, Grasset, Paris, 1993, 14. Voir ici même Chapitre 1. 
tance aggravante - la perte de maîtrise dont pâtit le mâle atteste d'un manque de «sang-froid» incompatible avec sa supériorité naturelle d'alors sur l'élément femelle de la société placé moins haut dans la hiérarchie des êtres du fait précisément de trop prêter aux sentiments, aux affects? Le mâle s'y révèle en effet passif - il pâtit de ses propres affects - et partant dégradé puisque se retrouvant dans la position la plus contraire à un modèle de virilité hérité des Romains

Dans cette espèce, l'Histoire aura eu garde de trancher: les droits pénaux ont consacré les deux solutions opposées, montrant que les émotions n'ont pas de place dans le droit mais que celui-ci leur fait une place, qu'elles sont de nature ancillaire, de l'ordre de la «circonstance» donc de la contingence et par là n'atteignent pas au cœur (essence) du droit. En bref, que le droit dispose des émotions selon ses propres réquisits.

Grandeur du droit, hauteur même, que la distance d'avec les choses, les sentiments communs, met à l'abri de quelque jugement vulgaire parce qu'empreint d'une subjectivité non dominée, d'une sentimentalité évidemment mauvaise conseillère, trop aisément sujette à donner dans l'arbitraire. Noble et classique image du droit, consonant avec la révérence dont jouissaient les gens de robe, aux échos lointains de sacralité.

Mais le réalisme américain, ramassé dans la formule «breakfast jurisprudence» ne dit-il pas tout l'inverse: que la mise à distance de l'espèce que réaliserait le magistrat n'est que naïveté idéaliste ou hypocrisie corporatiste ? Et que pour connaître l'intention voire les émotions du juge dans telle affaire - Vorverständnissen ou préjugements évidemment de première (et quasiment seule) importance pour le plaideur -, mieux vaut connaître le menu du petit déjeuner du président de la cour: sa confiture préférée ne lui manqua-t-elle pas? A-t-il bien digéré son café au lait ou siégera-t-il avec quelque aigreur d'estomac, quand ce ne serait pas d'âme? Sa fille n'a-t-elle pas été fauchée par un chauffard en état d'ébriété, «comme» le conducteur alcoolique dont il faut juger du comportement criminel ce matin?

Que conclure alors de ces deux positions diamétralement opposées en matière de crime passionnel: le juge esclave ou maître de ses émotions?

Nous sommes par là passé, presque subrepticement, des émotions dans le chef de l'auteur d'une action intéressant le droit au chef de qui rend la justice, le juge. L'assomption des émotions en droit s'annonce dès lors complexe et nous ne prétendrons guère à plus que suggérer quelque ordre en la manière d'une heuristique des affects en droit.

Aussi distinguerons-nous entre émotions «sous» le droit, émotions «dans » le droit et jusqu'aux émotions «au principe» du droit.

Pour bien comprendre l'assomption des émotions par le droit, il convient, d'un point de vue heuristique, d'avancer une position forte voire provocatrice pour mieux distinguer ces trois degrés de prise en compte: iram ou furiam non novit jus, le droit ne connaît pas la colère ou la furie, furie assumée ici comme parangon des émotions. Cela parce que le droit ne connaît rien que lui-même - d'où sa définition nécessairement tautologique: «toute tentative de définition, aussi brutale ou sophistiquée qu'elle soit, finit toujours par ajouter, en désespoir de cause, l'adjectif «juridique» pour le qualifier. Qu'on cherche à l'établir par la loi, par la règle, la sanction, l'autorité, le bien commun, le monopole de la violence, l'Etat, il faut à chaque fois préciser: «à condition qu'ils soient juridiquement fondés ». 
Autrement dit, une inévitable tautologie fait partie de la définition du droit. Pour décrire le droit de façon convaincante, il faut être déjà, par un saut, installé en lui.» ${ }^{3}$

Définition tautologique mais pas pour autant «circulaire» tenterons-nous de montrer, tout en évitant l'auto-référentialité, affirmation plus dogmatique qu'éclairante. A-t-on suffisamment dit les avantages et les enseignements profonds de la tautologie, par exemple qu'elle garde la pensée de la paresse intellectuelle consistant à poser des points-origine? Qu'elle interdit de refermer la réflexion à propos des fondements du droit, plus délicat encore du juste, s'ils ne se confondent pas? Qu'en cette béance réside la meilleure assurance de ne pas précipiter le droit vers une dogmatique - formalisme des apparences ou des valeurs -, entreprise des plus funestes pour une praxis c'est-à-dire un savoir, mieux savoirfaire (ars juris, art du droit), qui doit intégrer le contexte, toujours «droit en situation ${ }^{4}$, et que la réduction de son application à une simple mécanique de subsomption vient ruiner dans sa prétention (au sens positif) même à dire le juste «du» (dans et pour) cas singulier? ${ }^{5}$

Le droit s'affiche comme une branche, une activité humaine, dépourvue d'émotions, à tout le moins en apparence. La robe permet de les masquer, les rites judiciaires (et plus généralement les règles de procédures) de les diluer jusqu'à leur quasi effacement. Les complets noirs, gris ou bleus sombres des gens de justice participent de cette mise à distance de l'émotionnel. Quand bien même fûtil plaidé, l'émotionnel n'y est jamais qu'instrumentalisé, vidé de sa spontanéité: il s'agit d'un émotionnel rhétorique, ce qui ne signifie pas inexistant ou feint mais simplement non spontané, non immédiat, à la sincérité «calculée».

Mais s'il est besoin d'instrumentaliser l'émotionnel, à savoir de le réduire à un moyen, peut-être est-ce bien qu'il préoccupe gravement le droit, comme une menace permanente sur l'ordre (politico-juridique), qu'il sied de contenir en l'assumant au sein même de ce droit, manière de ressort demeuré implicite: "Sous une telle lumière [celle de la garantie divine en tant que l'on doit s'en passer et qui partant nous oblige à ne juger «qu'en tremblant»], il n'est pas jusqu'aux grands principes du droit qui n'apparaissent comme de simples recettes empiriques, conditionnées, limitées, par la nature humaine - des idées, rien de plus, et que l'on aurait tort d'ériger en idoles. L'égalité, par exemple, que de nos jours beaucoup regardent comme le sommaire de toute justice. Il ne faut pas en gratter bien longuement le vernis - le vernis des Bills of Rights et des Déclarations de droits pour en discerner par en-dessous d'atroces jalousies historiques. Pourtant, il n'est pas de moyen plus efficace d'éteindre la jalousie que de se montrer, entre les jaloux, résolument égalitaire. Et la légalité, donc [...] Entre la frénésie que les uns ont de commander, les autres de désobéir, c'est presque un coup de génie; que d'avoir imaginé ceci: que le commandement appartiendra, que l'obéissance sera due, à un ordre qui n'a point de visage - dont on peut attendre, puisqu'il est imper-

3 B. Latour, La Fabrique du droit, La Découverte, Paris, 2002, 273-274.

4 Voir par exemple A. Papaux, Introduction à la philosophie du «droit en situation», Bruylant/L.G.D.J/Schulthess, Bruxelles/Paris/Zurich, 2006.

5 Voir Chapitre 3 pour l'articulation entre le standard et la marge, celle-ci se disant à partir de celui-là. 
sonnel, qu'il interpellera sans passion et sera écouté sans colère.» ${ }^{6}$ Iram non novit jus disions-nous.

Il y a quelque chose du désir mimétique de René Girard au fondement même du droit moderne - et peut-être de tout droit, désir à atténuer, à apprivoiser pour que se perpétue la cité. Et comment mieux briser l'extrême singularité, jusqu'à l'idiosyncrasie, de l'émotion ressentie par tel particulier, dans telle circonstance ce qui le couperait de tous les autres et menacerait, potentiellement, le «vivreensemble» en son fondement moderne: la Raison - si ce n'est en la vidant de tout relief de valeur en en faisant un simple «fait» en et pour le droit?

Voilà les émotions réduites au statut de «fait», rejoignant en cela le sort que réserve le droit à toutes les données non juridiques que les réalités sociétales humaines lui «imposent» et dont il se sert pour dire le droit ${ }^{7}$, le juste («jurisdictio »), à savoir rendre justice dans et au cas singulier. Les données scientifiques ne bénéficient point d'un meilleur statut; et comme les émotions relèvent désormais pour partie de ces dernières, une esquisse des rapports entre droit et sciences - Chapitre 1 - nous permettra d'étudier une première manière de considérer juridiquement les émotions: les émotions «sous» le droit; le Chapitre 2 traitant, lui, des émotions «dans» le droit; et le Chapitre 3 des émotions «au principe» du droit comme nous le rappelions à l'instant par la plume du Doyen Carbonnier.

\section{CHAPITRE 1. \\ LES ÉMOTIONS «SOUS» LE DROIT : ESQUISSE DES RAPPORTS ENTRE SCIENCES ET DROIT OU LE DROIT COMME «PERTINENCE DE PERTINENCE»}

La frontière entre nature et culture, Objet et Sujet, s'étant en grande partie effacée, l'homme contemporain - par opposition au moderne dans la forme canonique du kantisme - se sait habiter un monde d'hybrides, rendant problématiques les relations entre sciences et droit, les plongeant dans une sorte de confusion. D'où la mise en garde de Latour: "Il devient urgent de ne pas demander aux sciences de trancher, de ne pas exiger du droit qu'il dise vrai.» ${ }^{8}$

Aussi banales qu'inexplorées en droit, les émotions ${ }^{9} \mathrm{n}$ 'y obtiennent guère plus que la place de «faits», dont la réception dans la sphère juridique n'est rien moins que passive. Les faits ne s'imposent pas au droit comme des donnés: il les «informe» selon ses propres besoins, à l'exemple des fictions juridiques, médiation active laissant entrevoir des rapports sciences/droit bien plus complexes qu'une obéissance du juriste (et singulièrement du juge) à la parole sacrée de l'expert

6 J. Carbonnier, Flexible droit. Pour une sociologie du droit sans rigueur, Paris, LGDJ, 8 ème éd., 1995, p. 441 (sous Codicille du juste et de l'injuste).

7 Sur ce point, voir notamment les contributions de P. Moor et A. Flückiger dans le présent volume.

8 B. Latour, op. cit., 257.

9 Comme thématique générale s'entend; des études particulières et détaillées existent, singulièrement en droit pénal et en droit de la procédure. On lira notamment l'article, fort bien documenté, de A. Flückiger dans cette revue. 
scientifique - dans le domaine des émotions : psychologue, psychiatre, psychanalyste, neurophysiologiste,... - drapé dans une objectivité quasi transcendante.

Encore faudra-t-il distinguer cette réception «valorisée» des faits en droit - en particulier les faits scientifiques ${ }^{10}$ - de l'extraction de valeurs sises «dans» ces faits ${ }^{11}$. La première constitue la trame même du droit: catégoriser et qualifier le «réel»- et donc en disposer, le manipuler - pour l'assumer dans un discours du «juste». L'extraction, elle, renvoie au paralogisme naturaliste (naturalistic fallacy) dont la formulation canonique interdit de déduire des faits des considérations de valeurs: on ne déduit pas le ought du is. Mille fois répétée, la cohérence de la formule - et non sa vérité, question en soi, nécessitant une discussion propre - s'avère plus que douteuse: le mode d'inférence top-down ou analytique de la déduction ne se prête pas à tirer quoi que ce soit du fait déjà, par définition, «down», sauf à passer d'un fait «complexe» à un fait «simple» - laissant alors dans l'ombre le saut qualitatif de la reconstruction systémique du tout à travers ses «parties » et notamment l'épineux problème des propriétés émergentes - ou du fait «générique» au fait «spécifique» - passage qui, cette fois-ci, laisse dans l'ombre la provenance de la «différence spécifique» pour le formuler dans le langage de la syllogistique.

Le droit n'est de toute façon pas concerné par la naturalistic fallacy si l'on accepte de le définir épistémologiquement «pertinence de pertinence» ou «pertinence ${ }^{2}$ », à savoir un point de vue sur les points de vue sur le réel, ces points de vue sur le réel lui étant fournis par tout ce qui est non-juridique. Les émotions relèvent de ce dernier domaine.

Le droit doit cette position originale de «pertinence ${ }^{2} \gg$ notamment à deux singularités: l'obligation de trancher d'une part ou interdiction du déni de justice; sa force instituante d'autre part, cette force instituante ne se confondant aucunement avec le performatif par lequel la théorie juridique souvent pense saisir la spécificité du droit. En effet, seul un contexte sémiotiquement approprié, à savoir institué à dessein et donc reconnaissable par les locuteurs, permet à telle ou telle formule langagière d'instaurer ou d' «acter» ce qu'elle «dit». Où le performatif apparaît comme un résultat, le fruit d'un contexte typique préparé par des social patterns sédimentés dans l'esprit des acteurs d'une culture ou société donnée. La réussite performative le doit donc à la sémiotique et non au langage et ses «actes».

Le juriste en face de faits les assume autrement qu'en se conformant à une logique déductive: il procède suivant une logique indiciaire, plus modeste, plus lâche, mais infiniment mieux adaptée à son travail. Le rationnel le cède alors au raisonnable, le droit cherchant à relier les indices (constitués notamment des «faits » à « in-former», parmi lesquels les émotions) dans une fabula, un récit qui puisse aboutir à une solution juste, un juste aussi bien singulier que collectif,

${ }^{10}$ Maintes notions juridiques sont pénétrées de dimensions scientifiques ou technico-scientifiques, éclairées par des experts avant que de prendre corps dans le droit: 1' «irresponsabilité» d'un accusé, la «gravité» d'une faute dans nombre d'activités couvertes par les assurances, le «manquement » aux règles de l'art dans diverses professions libérales notamment, etc.

${ }^{11} \mathrm{Si}$ pareille extraction est possible, ce que soutiennent certaines doctrines juridiques dont l'épistémologie fait encore fond sur l'adage res ipsa loquitur (la chose parle d'elle-même). 
partant, de l'ordre de l'acceptable, si finement décrit par Ricoeur: «la finalité courte de cet acte [de juger] est de trancher un conflit - c'est-à-dire mettre fin à l'incertitude -, sa finalité longue est de contribuer à la paix sociale, c'est-à-dire finalement à la consolidation de la société comme entreprise de coopération, à la faveur d'épreuves d'acceptabilité qui excèdent l'enceinte du tribunal et mettent en jeu l'auditoire universel si souvent évoqué par Ch. Perelman.» ${ }^{12}$

Confronté à cette logique indiciaire, d'aucuns crieront à un droit faible, subjectif, voire arbitraire. Semblable logique s'avère pourtant plus fréquente qu'on ne le croit sous la doxa scientifique encore largement positiviste, parfois même scientiste. Par exemple, en lien avec les émotions, diverses théories des «états mentaux» usent de cette logique, explicitement même, en affirmant au principe de leur recherche l'impossibilité d'un accès direct à ces états et donc le passage obligé par les indices. Sans doute les réflexions portant sur ce passage demeurent-elles fort rares, rarissimes mêmes, au regard de la luxuriance des écrits en affective sciences.

Indices, corrélations, causalités seulement plausibles, «causalité adéquate» selon la terminologie juridique helvétique par exemple, suffisent au juriste dans le cadre d'une fabula à reconstruire selon le vraisemblable seulement. Où l'on retrouve une conception classique du droit comme rhétorique, laquelle ne doit aucunement être rabattue sur quelque élégance de parole. Le droit s'éprouve principiellement rhétorique parce qu'il doit aller au particulier en passant par le genre - il y a des lois, générales et abstraites, à respecter - tout en le dépassant dans la mesure où il cherche à rendre justice au et dans le cas singulier, le «in casu» ou encore le «in concreto» dans le langage juridique.

Comment pourrait-il en aller autrement de cette praxis humaine toute pénétrée de langue, de code: «Il est clair que l'acte de parler, même quand il implique la subordination de ce qui est actuellement visé à la généralité d'une signification préalablement donnée dans un mot, ne peut pas être conçu comme la combinaison d'actes de subsomption qui subordonnent chaque fois quelque chose de particulier à un concept général. Celui qui parle, c'est-à-dire qui emploie des significations de mots qui sont générales, est tourné d'une façon telle vers ce qu'a de particulier une intuition portant sur la chose même, que tout ce qu'il dit participe à la particularité des circonstances qu'il a en vue.» ${ }^{13}$

Où l'on retrouve le jeu sémiotique entre Contenu Nucléaire, Type cognitif et Contenu Molaire: «Le Contenu Nucléaire, ainsi que le Type Cognitif qu'il interprète, ne représente pas tout ce que nous savons sur une unité de contenu donnée [unité sémantique]. Il représente les notions minimales, les qualités requises élémentaires pour reconnaître un objet donné ou comprendre un concept donné et comprendre l'expression linguistique correspondante» selon Eco $^{14}$. Le sémioticien italien précise: «Au niveau de Contenu Nucléaire, il devrait y avoir un

12 P. Ricoeur, Le Juste, Editions Esprit, Paris 1995, 10. L' « auditoire universel » est «constitué par l'humanité tout entière, ou du moins par tous les hommes adultes et normaux» (Ch. Perelman et L. Olbrechts-Tyteca, Traité de l'argumentation, Editions de l'Université de Bruxelles, 5 'ème édition, Bruxelles, 1988, 39).

${ }^{13}$ H.-G. Gadamer, Vérité et Méthode, Seuil, Paris, 452.

${ }^{14}$ U. Eco, Dire presque la même chose, Paris, Grasset, 2006, 104. 
consensus généralisé, fût-ce avec quelques effrangements et zones d'ombre, tandis que le Contenu Molaire, qui prend des formats divers selon les sujets, représente un vaste ensemble de compétences sectorielles. Disons que la somme des Contenus Molaires s'identifie avec l'Encyclopédie en tant qu'idée régulatrice et postulat sémiotique $»^{15}$.

D'un point de vue pragmatique, le Contenu Nucléaire ne cesse de poser problème. Partant, l'aborder par les Contenus Molaires, nécessairement sectoriels et même personnels, complexifie fortement sa circonscription, particulièrement en matière judiciaire, et de par la diversité des acteurs judiciaires impliqués donc de leur Contenu Molaire et de par la domination (réelle ou supposée) de la figure de la reconnaissance. Par définition, en effet, le juge ne peut que reconnaître et non connaître le Contenu Nucléaire, à tout le moins dans un système formalistelégaliste érigeant en absolu la séparation des pouvoirs, le «connaître » revenant évidemment au législateur. D'où, en pratique, la prépondérance du raisonnement par analogie en droit, dans la conception légaliste déjà, et éminemment dans une conception du juridique comme «droit en situation». Cette primauté interdit au reste de réduire l'opération de reconnaissance à une subsomption sur le modèle du positivisme légaliste: des dissemblances (entre le Type et l'occurrence notamment) sont toujours présentes, fussent-elles habilement narcotisées par l'interprète. En d'autres termes, le nucléaire légal (tel que livré dans la loi) opère seulement à la manière d'un attracteur, l'opération de qualification-catégorisation juridique pouvant de la sorte assumer les effrangements et petits déplacements des occurrences par rapport au Type ; en ce point sourd l'abduction, seule congrue à des catégories juridiques conçues selon un mode matriciel, et mnémonique verrons-nous (Chapitre 3).

Le poids des circonstances invalide toute tentative d'explication de l'application du droit par la seule logique de la subsomption. L'inévitable médiation du contexte réaffirme la force d'un particulier qui ne s'épuise pas dans le général. $\mathrm{Au}$ rebours, le particulier en droit excède son «genre» tout en y participant. De là un «genre» en mouvement relèvera Gadamer: "Mais cela signifie, inversement, que le concept général visé par la conceptualisation ne cesse pas de s'enrichir luimême à la faveur de ces intuitions de la chose, de sorte qu'il finit par en naître parfois un mot nouveau et plus spécifique, qui rend mieux justice au caractère particulier de cette intuition de chose. Par conséquent, bien que l'acte de parler présuppose nécessairement l'usage de mots préalablement donnés et dotés de leur signification générale, il y a tout de même place pour un processus constant de conceptualisation, grâce auquel la vie sémantique de la langue même continue à se développer.» ${ }^{16}$

La langue se révèle donc bien davantage matrice que substance ou dictionnaire ou code ; une marge de variation appartient intrinsèquement à ses « unités séman-

${ }^{15}$ Idem, 106. Sur la notion d'encyclopédie, voir U. Eco, Sémiotique et philosophie du langage, Paris PUF, 1988, passim. Sur la distinction entre «encyclopédie» et «dictionnaire» appliquée au droit, voir notamment A. Papaux, «Un modèle dynamique de catégorisation juridique: l'encyclopédie sémiotique de U. Eco» in International Journal for the Semiotics of Law, Chicago, Vol. 17, No 1, 2004, 1-25

${ }^{16}$ Gadamer, op. cit., 452. 
tiques ». Plus on va dans le détail de l'occurrence, plus la variation s'affirme, de la singularité jusqu'à l'idiosyncrasie. On comprend alors les enjeux formidables du droit, authentique quadrature du cercle: dire le juste dans le cas, prenant en compte ses singularités les plus intimes, tout en passant par le texte d'une norme (loi) par définition générale et abstraite. Le «genre» doit alors sauter - saut qualitatif - jusqu'au singulier, lequel doit à son tour en participer pour bénéficier de la légitimité dont la loi est revêtue de par son origine démocratique. Compte tenu de ce saut qualitatif, et de l'impossibilité subséquente d'y répondre par de simples moyens de logiques formelles, ainsi que de l'obligation de rendre justice et de rendre justice au cas et dans le cas, le droit ne peut se passer de la rhétorique et prend grand soin de justifier ses solutions par la publication des jugements et l'obligation corrélative de les motiver.

Le droit s'avère de la sorte substantiellement rhétorique, comme nous le rappelions, mais selon une acception exigeante de la rhétorique, proprement cognitive: «Entre les réponses inexistantes pour des problèmes qui sont eux-mêmes objets d'interprétation et de discussion (genre délibératif) et celles purement conventionnelles pour des problèmes qui n'en sont presque plus (genre épidictique) on trouve les problèmes bien réels qui divisent les hommes mais pour lesquels il faut trouver et exercer le bon jugement, le juste jugement (genre judiciaire). Le droit est ainsi la mise en œuvre de l'identité et la différence entre les hommes au sein d'un nouveau $\log o s$ qui en régule les modalités. Un tel $\log o s$ est forcément une rhétorique, le discours de la rencontre des hommes et du langage..${ }^{17}$

On comprend dès lors mieux la liberté voire la distance que prend le droit visà-vis des sciences et par là des faits dans leur forme la mieux assurée, celle des protocoles, de la répétabilité. Ce point de vue en surplomb du droit, parce qu'il recherche le juste et pas nécessairement le vrai, permet au juriste (particulièrement au juge) de démêler, sur le seul plan juridique évidemment, des situations demeurant profondément contriquées dans le champ scientifique. Le principe de précaution se contente par exemple de plausibilité, donc en l'état de corrélations, pouvant donner lieu à «responsabilité» juridique alors que la causalité scientifique liant l'activité et le dommage potentiel ou actuel n'a pas été mise à jour. Dans une veine analogue, des Etats fédérés américains ont relevé l'âge seuil pour l'obtention du permis de circulation suite à des données d'imagerie cérébrale révélant que les aires activées lors de la conduite automobile ne sont pas les mêmes chez les adultes et chez les jeunes gens entre 16 et 25 ans environ, la prise de risque étant naturellement plus importante concernant les derniers sujets ${ }^{18}$.

${ }^{17}$ M. Meyer, Qu'est-ce que la philosophie?, Paris, Le livre de poche, 1997, 173. On pensera évidemment à Aristote, Les Politiques, I, 2, 1253 a (traduction Pellegrin, GF-Flammarion, Paris, 1993, 91-92): «seul parmi les animaux l'homme a un langage [... lequel] existe en vue de manifester l'avantageux et le nuisible, et par suite aussi le juste et l'injuste. Il n'y a en effet qu'une chose qui soit propre aux hommes par rapport aux autres animaux : le fait que seuls ils aient la perception du bien et du mal, du juste, de l'injuste et des autres < notions de ce genre>. Or avoir de telles < notions $>$ en commun c'est ce qui fait une famille et une cité.»

${ }^{18}$ Pour des références et de plus amples développements et d'autres exemples analogues, voir notamment A. Papaux, «Détermination biologique des comportements et responsabilité individuelle. Une approche de philosophie du droit», in Morale et évolution biologique: entre déterminisme et liberté, Presses Polytechniques et Universitaires Romandes, juin 2007, 295 ss. 
Mais dans tous les cas le juriste demeure le maître du choix, de la pertinence, de ce qu'il adviendra de tel fait en l'orbe juridique. Où l'on retrouve le droit comme «pertinence ${ }^{2} »$ (pertinence de pertinence). Cette position de surplomb ne l'expose pas moins au débordement des émotions, à son éventuelle submersion par le torrent des affects; pour éviter d'en pâtir, le droit, plus pragmatiquement le «dire-le-droit», s'est toujours coulé dans des procédures (Chapitre 2), droit dit «formel» (par contraste avec «matériel» ou «substantiel»): il faut bien de la tenue pour pratiquer le droit.

\section{CHAPITRE 2. LES ÉMOTIONS «DANS» LE DROIT : CACHEZ CE SEIN QUE NOUS NE SAURIONS VOIR}

Si les émotions sont «sous» le droit tel que l'on vient de le définir, on en trouve également dans le droit, non comme «faits» pour le droit mais comme force le mouvant, menaçant parfois de le faire tomber dans l'arbitraire. Les stratégies de mise à distance des émotions, autres que celles rentrant dans les social patterns des fabula à construire selon la logique indiciaire, sont classiques. Elles relèvent principalement de la procédure (juridique): modes de preuve prédéterminés et limités, composition collégiale des tribunaux, voies de recours nombreuses, obligation de motiver, etc.

Il n'est pas jusqu'au syllogisme qui ne tente d'assumer les émotions dans le droit, malgré son caractère logique formel: «Le syllogisme [...] est peut-être surtout un moyen par lequel le juge tente d'apaiser les parties par une apparence de généralité et de clôture.» ${ }^{19}$ Pris dans la distinction entre l'Effectuation du droit et sa Présentation ou mise en formes a posteriori, on voit que le positivisme ne se réduit en rien à quelque intention de manipulation des faibles ou des gouvernés par une caste de juristes toute préoccupée au maintien de ses prestige et avantages mais recherche l'apaisement collectif en imprimant les marques d'une parole définitive, seule à pouvoir porter la paix sociale. On lit trop facilement l'hypocrisie derrière ou sous les formes du syllogisme dans lesquelles on reconstruit ou Présente le raisonnement juridique Effectué de manière non déductive; il s'agit bien davantage de l'assomption, pour les canaliser, d'émotions collectives, lesquelles, ainsi « régularisées » (de regula, la règle en latin), ou seulement contenues, participent à l'érection de la cité plutôt qu'à son incessante remise en cause voire destruction.

C'est dire que le droit prête la meilleure attention aux émotions et plus généralement aux «faits». Affirmation si vraie ou pertinente que: «Le Droit ne doit jamais oublier que ses conceptions ne sont valables que dans la mesure où elles sont suffisamment ajustées aux faits qu'il doit régir et aux besoins et aspirations que la vie en société vise à satisfaire ${ }^{20}$, étant rappelé que ces ajustements

19 S. Mc Evoy, «La question de l'arrêt: le cas de l'argumentation dans le droit», in Lire le droit. Langue, texte, cognition sous la direction de D. Bourcier et P. Mackay, LGDJ, Paris, 1992, 178.

${ }^{20}$ L. Husson, Nouvelles études sur la pensée juridique, Dalloz, Paris, 1974, 232, où l'on retrouve en filigrane la notion du degré d'anticipation, plus globalement celle de la commensurabilité de la loi aux cas. 
sont toujours opérés à sa manière, à sa convenance, fonction de ses propres finalités.

L'analyse du droit sous l'angle formel ou procédural ne retiendra pas notre attention au-delà de ce bref rappel de quelques principes ou figures juridiques exprimant la mise à distance des émotions menaçant l'objectivité ou la rationalité du «dire-le-droit».

Infiniment plus délicates à analyser les émotions «au principe» du droit: elles engagent toute l'entreprise du droit (moderne à tout le moins) dans la mesure où elles pourraient en constituer le fondement, fût-ce en creux : ce contre quoi le droit n'a eu de cesse de lutter et qui a abouti aux systèmes juridiques.

\section{CHAPITRE 3. \\ LES ÉMOTIONS «AU PRINCIPE» DU DROIT : LE DROIT COMME SENTIMENT?}

Avec le droit comme sentiment, on mesure toute l'importance de la distinction entre sentiment et émotion, s'il y a lieu d'en établir une ${ }^{21}$. Quoi qu'il en soit, posant la question de manière générique, nous nous demanderons si des émotions, sous la dénomination de «sentiment», se trouvent au fondement du juridique.

Nous parcourrons cette troisième instance de la présence des émotions en droit, après les émotions «sous » et «dans » le droit, en esquissant les émotions au principe du droit dans le chef de l'équité singulièrement ou, corrélativement, de l'arbitraire sous le mode de son interdiction, lequel condamne tout comportement ou activité contrevenant «au sentiment de la justice et du droit» tel qu'il existe dans une communauté nationale donnée ${ }^{22}$, selon une formule partagée dans son esprit par les ordres juridiques continentaux.

La caractérisation de l'équité «Sentiment sûr et spontané du juste et de l'injuste; en tant surtout qu'il se manifeste dans l'appréciation d'un cas concret et particulier $\gg^{23}$ exprime une dimension cognitive (comme la rhétorique, supra Chapitre 1) du sentiment, qualifié à ce titre de «sûr».

Le renvoi qu'opère Salmon dans son Dictionnaire de droit international public d'Equité à Raisonnable permet de mettre en exergue un jeu complexe de singularité et de standard, se développant entre l'assomption du contexte ${ }^{24}$ contribuant éminemment à la singularité du cas et l'affirmation d'une norme-normalité, d'un standard, ce qui confirme indirectement qu'en droit est considérée la maîtrise de l'émotion bien davantage que l'émotion elle-même.

${ }^{21}$ Pour une discussion de cette distinction, voir notamment dans cette revue les différents articles de biologistes, psychologues et philosophes des émotions.

${ }^{22}$ Pour la Confédération Helvétique, voir par exemple la revue Semaine Judiciaire 2006, ${ }^{\circ} 31$, p. 424.

23 A. Lalande, Dictionnaire technique et critique de la philosophie, PUF, Paris 1996, 295.

${ }^{24} \mathrm{~L}$ '«encyclopédie» au sens de la sémiotique et donc de la pragmatique, par contraste avec le «dictionnaire», contient, à tout le moins cherche à saisir, les marques d'insertion contextuelle des «mots»; sur ces notions, voir les travaux de U. Eco. 
En témoigne explicitement l'entrée Raisonnable dans ce même dictionnaire. On y lit: «adjectif qualifiant un acte ou un comportement approprié à toutes les circonstances pertinentes de l'espèce.» Suit une citation de la Cour internationale de justice. Puis: «En ce sens, «raisonnable» s'apparente à ce qu'il est normal de faire, dans des circonstances similaires à celles de l'espèce; il renvoie à un standard de comportement.» Suit la citation d'une sentence arbitrale. Puis: «Le terme s'oppose à ce qui est excessif, exagéré; s'apparente à ce qui est modéré, ordinaire.» Affirmé ensuite plus large que l'adjectif Equitable, lequel est davantage lié à la notion d'égalité, «raisonnable» ne saurait toutefois se passer de certaines considérations d'égalité, permettant à Salmon de conclure: ««raisonnable» se distingue ainsi de «rationnel $» .{ }^{25}$ De surcrôtt, un lien avec le but légitime poursuivi complète la définition du raisonnable.

L'équité apparaît donc bien comme un sentiment réglé, normalisé, sentiment cognitif même, mais éduqué à l'assomption du particulier, formé à la logique de l' «entre-deux » ou du passage du Type à l'Occurrence, souvent sur base de réminiscence d'occurrences précédentes oeuvrant comme prototypes ou premiers analogués. Le premier analogué s'entend du centre de gravité de la catégorie ou Type, qui en dessine le champ d'attraction, dont les forces opèrent selon l'intensité des similitudes rapprochant l'occurrence à catégoriser ou à qualifier de l'occurrence prototypique. En termes méthodologico-épistémologiques, «il n'y a plus en jeu de pures identifications ou absorptions (d'espèce à genre) mais soit des «similarités » soit des «oppositions » ${ }^{26}$. Le premier analogué ou premier connu à partir duquel se construit les ressemblances et s'apprivoisent les occurrences étranges et étrangères se donne comme entité concrète, raison pour laquelle le raisonnement juridique s'incarne en un (en vérité plusieurs) jugement estimant puis arrêtant, en fonction notamment du contexte - puissant instrument de désambiguïsation $^{27}$ - une primauté des ressemblances. Cette mimésis s'établit suivant des appréciations gradualistes et non conformément au syllogisme dont la majeure contiendrait un nombre fini de traits sémiologiques ou sémantiques ultimes (dénommés primitifs), imperméables à tout contexte, que la mineure doit épouser trait pour trait dans une logique du «conforme / non-conforme». La démarche syllogistique, pétrie de constatations abstraites et binaires, vide l'équité de toute pertinence, au rebours du jugement élaboré sur base d'un prototype ou du premier analogué, jugement de proportionnalité et de ressemblances (et dissemblances) qui contient, au moins implicitement, l'application de l'équité. Un déplacement s'opère de la sorte du rationnel au raisonnable.

L'entrée Equité des Notions philosophiques ${ }^{28}$ apportera des éléments précieux dans le cadre de l'examen du fondement (éventuel) du droit dans les émotions ou les sentiments. Nous pensons à deux relectures critiques des théories des émotions

${ }^{25}$ Dictionnaire de droit international public (sous la direction de J. Salmon), Bruylant, Bruxelles, 2001, 923-924 pour Raisonnable et 441-443 pour Equité.

${ }^{26}$ Eco, Sémiotique, 151.

${ }^{27}$ Eco, Dire presque la même chose, en particulier, et pour cause, la section «Comprendre les contextes» $(33 \mathrm{ss}$.)

${ }^{28}$ Notions philosophiques, Encyclopédie philosophique universelle, 2 tomes, sous la direction de S. Auroux, PUF, Paris, 1990, 822 ss. 
synthétisées par Livet dans le document préparatoire du colloque dont sont tirées les contributions de ce numéro de revue, et intitulé: "Rationalité et émotions: examen critique». La première relecture s'entend de la seconde voie d'étude parmi les trois relevées par Livet, à savoir la perspective du raisonnable: assomption des émotions en tant qu'elles correspondent à l'idée du raisonnable, et par là position d'une rationalité limitée mais capable de progrès. La seconde relecture correspond à la troisième voie d'étude, celle de la rationalité relative et donc de la coexistence entre les valeurs - question de position relative des unes par rapport aux autres.

Sous l'entrée Equité, nous trouvons deux divisions principales, aux éléments saillants suivants, pointant vers l'une ou l'autre «relativisation» de la raison (ou Raison) sous l'effet de la prise en compte des émotions dans la notion contemporaine de rationalité :

A) L'équité, reflet de la nature des choses: identité entre justice et équité chez Aristote et Thomas d'Aquin; l'équité «assure le passage du rationnel au réel», elle est «l'accomplissement de la justice légale.» Il siéra lors tenir compte des circonstances. A la médiation entre les prétentions adverses, l'équité ajoute «une médiation entre les critères à suivre ${ }^{29}$. La «nature des choses » est ici déterminante, mais simultanément cette nature ne se donne pas simplement puisqu'il est renvoyé à la Rhétorique d'Aristote. Comme amélioration de la justice (laquelle serait simple application de la loi au cas), «l'équitable est le résultat de l'harmonisation des devoirs de justice avec les autres devoirs » (J. Hervada), suivant une lecture dite réaliste du monde, pourtant découlant de «l'intuition noétique de la nature de cette chose». Quoi qu'il en soit, transparaissent ici des éléments de la «rationalité relative».

B) L'équité, exigence de la conscience: perspective moderne ménageant à l'équité une simple fonction supplétive, remédier aux lacunes d'un texte (normatif) et répondant «à un appel de la conscience du juge et émane irrationnellement de son for intérieur» (F. Gény cité en référence). L'équité a alors la personne pour objet et pour fin justificative.

On rappellera qu'il s'agit de la personne moderne dont l'essentielle propriété réside en la Raison, jetant de la sorte bien de la suspicion sur cette équité, trop pragmatique, trop souple, trop sensible aux contextes, pour ne pas apparaître, en effet, le fruit de l'irrationnel. Aussi l'équité sera-t-elle tenue à bonne distance de la loi au sein de la théorie juridique moderne dite "positivisme légaliste».

Or, c'est précisément cette Raison désincarnée, propre d'un Sujet transcendantal donc non «mondain» (au sens positif du terme), que les études sur les

${ }^{29}$ Rappelons, à ce titre, la subtile remarque de R. Brague, La sagesse du monde, Paris, Fayard, 1999, 173: «en bon aristotélicien, la sagesse (phronèsis) est une vertu dianoétique non pas éthique. C'est elle qui fournit aux vertus éthiques le logos qui leur permet d'atteindre le juste milieu.»; le «juste milieu » constituant la position éthique canonique de la pragmatique de l'action, des vertus, dans la philosophie d'Aristote. Il correspond à un optimum (fonction du contexte et de la personne) entre deux pôles impropres à réaliser pleinement le sujet, à l' «achever» selon l'entéléchie. 
émotions voire les affective sciences viennent relativiser dans le sens de son incarnation et par là d'un lien intime, intrinsèque, avec les émotions. Par où la Raison - désormais «raison» avec une minuscule - recouvre l'humilité qui fut toujours son propre dans les pensées d'obédience inductiviste ou pragmatique - «pragmatique » dans le sens d'élaborée à partir de la praxis, des existants (ou pragmata en grec) - sous les espèces du raisonnable.

L'équité en droit procède bien du raisonnable, n'apparaissant irrationnelle qu'aux yeux des théoriciens concevant le juridique comme éminemment abstrait. Le reflète la forme canonique du droit moderne, la loi, par définition générale et abstraite, et fruit de la Raison. La pratique du droit - l'ars juris (art juridique) n'a jamais emprunté semblable voie, comme l'a rappelé le Tribunal fédéral par contraste avec maintes doctrines encore travaillées par le mythe de la Rechtswissenschaft: quand bien même disposerait-on de méthodes (au sens fort, scientifique du terme), a fortiori usant de celle ménageant le moins - voire aucune liberté à l'interprète, soit la méthode d'interprétation dite littérale-grammaticale, le juge ne rend pas pour autant une décision «en» droit mais seulement «légale», légalité qui ne peut être maintenue que si elle exprime quelque juste. Le Tribunal fédéral affirmera en effet que la méthode grammaticale n'est soutenable que si elle conduit à un résultat matériellement juste, «droit»: «zweifelsfrei eine sachlich richtige Lösung ergab» ${ }^{30}$.

Ce n'est pas seulement la transformation de la Raison en «rationalité relative» (la troisième voie mentionnée par Livet) qui se trouve par là confirmée mais plus fondamentalement l'équité, ce sentiment du juste, comme ressort même du droit voire son essence (ou ultime instance). Que, partant, la loi n'en constitue qu'une modalité c'est-à-dire une expression de la «chose» (ou essence) et non la chose même. Le Tribunal fédéral nous engage donc lucidement à ne pas confondre le moyen et la fin, indistinction caractéristique du légalisme: le droit ne vise pas à la loi mais au juste, au «sachlich richtig», qu'il tente d'atteindre par le moyen de la loi, notamment, la sachant au reste intrinsèquement insuffisante ${ }^{31}$, ce qui ne le troublera nullement puisqu'elle ne constitue que l'un des ses modes d'expression, fût-il privilégié.

D'aucuns craindront que le droit ne s'abîme alors dans l'arbitraire, le bon vouloir du juge, crainte pertinente pour qui se tient dans un modèle légaliste pyramidal du juridique, lequel manque triplement au moins la valeur du cas (en droit).

En premier lieu, en ce que l'application n'est pas ancillaire par rapport à la loi mais tout au contraire l'achève, la «par-fait» au sens littéral: la fait être de part en part, la réalise dans toutes ses potentialités pour et dans le cas singulier, et cela depuis toujours.

30 Arrêt du Tribunal fédéral suisse 124 III 199, notamment.

31 «la loi est toujours quelque chose de général, et [qu'] il y a des cas d'espèce pour lesquels il n'est pas possible de poser un énoncé général qui s'y applique avec rectitude. Dans les matières, donc, où on doit nécessairement se borner à des généralités et où il est impossible de le faire correctement, la loi ne prend en considération que les cas les plus fréquents [...]. La loi n'en est pas moins sans reproche, car la faute n'est pas à la loi, ni au législateur, mais tient à la nature des choses, puisque par leur essence même la matière des choses de l'ordre pratique revêt ce caractère d'irrégularité.» (Aristote, l'Ethique à Nicomaque, V, 14 (267 de la traduction de J. Tricot, Vrin, Paris, 1970). 
En second lieu, de par ce fait, évident, que le droit ne s'épuise pas dans le cas, quand bien même l'occurrence s'éprouve-t-elle indispensable à sa perfection, à son achèvement. En ce sens, la loi n'est qu'un raccourci cognitif et mnémonique du droit à l'analogue de la théorie formalisée pour la physique ${ }^{32}$. Le droit parle donc bien au-delà de la loi à la faveur du cas. Le «dans le cas », se double en effet toujours d'un «à l'occasion du cas », ce dernier pointant non seulement vers le bien commun, nous rappelait Ricoeur ${ }^{33}$, la paix sociale, mais encore vers le renforcement ou l'infléchissement de l'ordre juridique lui-même, orienté par la publication plus ou moins étendue des décisions de justice, élisant de la sorte tel cas au rang de prototype ou expression exemplaire de la fabula (social pattern) arrêtée par le législateur dans la loi, découvrant la dimension pédagogique du droit, en éducation du sentiment du juste afin qu'il devienne plus «sûr» (supra) et plus «partagé».

Enfin, en troisième lieu, la logique d'insertion de la décision dans le tissu ou réseau d'une extrême densité que représente tout ordre juridique élaboré (et de quelque durée), rend illusoire la crainte de l'arbitraire (sauf ponctuellement, il va de soi) et parfaitement légitime, désirable et explicable, la subjectivité du juge, les prétentions à son objectivité formulées par la Rechtswissenschaft ayant été rendues caduques depuis l'avènement de l'herméneutique et du linguistic turn au moins. La logique d'insertion est si présente, si active, qu'elle exaspère les nonjuristes ainsi que nous le rappelle Latour: ««Nous sommes d'abord préoccupés de stabilité, nous devons tracer les sillons les plus droits possibles et les plus profonds, car le justiciable attend la cohérence et la visibilité.» [un Conseiller d'Etat français] Tous les traits des juristes qui énervent tant le sens commun, leur lenteur, leur goût de la tradition, leur esprit parfois réactionnaire, mais c'est leur fonction même: comme les Parques, le droit tient dans sa main le fil ténu de la totalité des jugements, des textes et des précédents qu'aucun accroc ne doit venir déchirer sous peine de déni de justice. Alors que le savant peut se satisfaire d'une information partielle parce qu'il sait que le pouvoir de ses instruments permettra à d'autres, plus tard, d'affiner le savoir et d'allonger les chaînes référentielles, le juge doit s'assurer que les trous sont ravaudés maintenant, les déchirures aussitôt rapiécées, les vides comblés, les affaires résolues, les arrêts rendus, les querelles éteintes. Si le tissu des sciences s'étend partout mais peut être partout vide, comme une dentelle, celui du droit doit tout couvrir, sans hiatus, sinon sans couture. Deux façons, totalement différentes, de couvrir le monde $»^{34}$.

L'équité offre une synthèse remarquable du raisonnable et de la rationalité relative. Du raisonnable d'une part, comme réincarnation de la Raison ou si l'on préfère l'affirmation pragmatique d'une liberté phénoménale et pas simplement nouménale pour reprendre le langage de Kant. De la rationalité relative, d'autre part, parce que le droit est éminemment pratique, «phénoménal», «relatif» puisque toujours non seulement dans une relation mais relation lui-même -

\footnotetext{
${ }^{32}$ On lira notamment le magnifique article de H. Volken, «Mnémosyne érudite. La théorie comme mémoire», Revue européenne des sciences sociales, Tome XLIII, 2005 ( $\left.\mathrm{N}^{\circ} 131\right)$, pp. 35 ss.

33 Voir la référence à P. Ricoeur, supra note 12.

${ }^{34}$ B. Latour, La Fabrique du droit, La Découverte, Paris, 2002, 258.
} 
comme le révèle le «test Robinson $»^{35}$ - et pour le moins selon une double dimension: le droit lie toujours deux sujets de droit, se situe «entre» eux, et il lie le standard et la singularité (supra).

Si le droit moderne se dit principalement dans (la forme de) la loi, il s'achève principiellement dans le cas, se développant du juste sédimenté au juste vivant, mû par l'équité, sentiment du bien et du mal que les hommes, vivants politiques (zoon politikon) à savoir constituant leur identité au sein de la cité, ont en partage, du fait même de leur pratique du collectif.

Un sentiment plus ou moins commun, plus ou moins éduqué, cognitif assurément, «mais »... un sentiment.

\section{Université de Lausanne}

${ }^{35}$ Par une Denkexperiment, imaginons Robinson, seul sur son île, plus dramatique encore seul au monde; en tant qu'occurrence de l'essence «homme», il bénéficie ipso facto de l'ensemble des droits de l'homme tels que reconnus dans les catalogues internationaux dont le plus efficace est sans conteste la Convention européenne des droits de l'homme. Mais que fera le pauvre Robinson avec ses libertés d'expression, de réunion, de religion, plus romantique, de mariage? Non que ces droits de l'homme deviendraient inutiles mais plus prosaïquement se trouveraient dépourvus de toute pertinence et même de toute signification. Cette disparition du sens donne à comprendre que le droit, contrairement aux habitudes de pensée modernes, est fondamentalement relationnel: sa substance est la relation, la présence (potentiel ou actuel) d'autrui, d'une altérité «de», «à partir» de, laquelle le droit reçoit sa signification. 